\title{
Effect of specimen size on cancellous bone microstructural parameters
}

\author{
K. Tanaka ${ }^{1}$, S. Sezaki ${ }^{1}$, S. Enoki ${ }^{2} \&$ T. Katayama ${ }^{1}$ \\ ${ }^{I}$ Department of Biomedical Engineering, Doshisha University, Japan \\ ${ }^{2}$ Department of Mechanical Engineering, \\ Nara National College of Technology, Japan
}

\begin{abstract}
As the average life span of Japanese people continues to extend, the increasing incidence of osteoporosis within the aging society is becoming a major public health problem. Such diseases have a negative impact on Quality of Life and an urgent issue to find prevention and treatment methods. Currently, correlation between the microstructural parameters and the mechanical properties is shown by using an X-ray microtomography. In this study, the influence of the cancellous bone region on the correlation between the microstructural parameters, calculated by using X-ray microtomography images, and Young's modulus, which were obtained from the compression test, was clarified by targeting cancellous bone specimens of various regions, obtained from bovine femur. Cancellous bone specimens were obtained from the right femur of a bovine for the compression test. The correlation coefficient between Young's modulus and microstructural parameters was calculated. Cubic specimen in $5 \mathrm{~mm}$ had the highest correlation coefficient between Young's modulus and microstructural parameters. Additionally, when specimen regions were 4 or $5 \mathrm{~mm}$, the standard deviation of each microstructural parameter had the smallest value, except for Trabecular Separation and Mean Intercept Length 3. The cancellous bone region of the cubic specimen in $5 \mathrm{~mm}$ had a high correlation between microstructural parameters and Young's modulus and the value of the microstructural parameters of the small variation.
\end{abstract}

Keywords: cancellous bone, X-ray $\mu C T$, microstructural parameters, compression test, specimen region. 


\section{Introduction}

As the average life span of Japanese people continues to extend, the increasing incidence of osteoporosis within the aging society is becoming a major public health problem. Osteoporosis decreases bone strength and increases the risk of fracture. Such diseases have a negative impact on Quality of Life, making it an urgent issue to find prevention and treatment methods.

Bone has a hierarchical structure and consists of the two parts, which are the cortical bone and cancellous bone. Since cancellous bone is metabolically more active than the cortical bone and its clinical condition easily appears, the density of cancellous bone is used for diagnosis of osteoporosis. Diagnosis of osteoporosis is made by various methods such as measurements of bone mineral density (BMD), dual-energy X-ray absorptiometry (DXA), quantitative X-ray computed tomography (QCT) and others $[1,2]$.

However, as was pointed out in the announcement from the National Institute of Health (NIH) in 2000, as an index showing bone strength other than bone density, the grope of the other "bone quality" parameters is important $[3,4]$. Bone quality refers to the characteristics that affect the function of bones to withstand fractures, other than bone density, and parameters include the trabecular structure, elastic properties, and bone turnover. In addition, the development of image diagnosis apparatus such as X-ray computed tomograph equipment enabled the shooting of finer microstructure of cancellous bone. From then on, study to evaluate the microstructure and mechanical properties of cancellous bone has become possible [5-7].

Currently, various studies have been conducted to evaluate the microstructure and the mechanical properties of cancellous bone in various ways such as compression tests. On the other hand, the micro-structural parameters of cancellous bone are calculated by using an X-ray microtomography and then calculated elements such as trabecular number (Tb.N), thickness (Tb.Th) and others were evaluated $[8,9]$. Moreover correlation between the microstructural parameters and the mechanical properties was shown there $[5,6]$.

On the other hand, cancellous bone is a heterogeneous material because it is a biological material. Nevertheless, correlation is evaluated by using the average value of the calculated microstructural parameters [7, 8]. Therefore, the cancellous bone area becomes large, variations in the microstructural parameters increase [5].

Although differences in cancellous bone region are considered to affect the mechanical properties $[10,11]$, effects of the cancellous bone region on microstructural parameters have not been taken into consideration. Therefore, by evaluating the effect of differences in the cancellous bone region on the microstructural parameters, it is deemed possible to reduce the variation in microstructural parameters and to improve the correlation between the microstructural parameters and mechanical properties.

In this study, the influence of the cancellous bone region on the correlation between the microstructural parameters, which were calculated by using X-ray microtomography images, and Young's modulus, which were obtained from the 
compression test, was clarified by targeting cancellous bone specimens of various regions which, were obtained from bovine femur.

\section{Preparing specimens and experimental procedure}

\subsection{Materials}

Cancellous bone specimens were obtained from the right femur of a bovine (at the age of 26 months) for the compression test. As shown in Fig. 1, cancellous bone specimens were shaped in a $10 \mathrm{~mm}$ cubic geometry by using a band saw and a sanding sheet. Specimens' axes were defined as follows: $Z$ axis is parallel to femoral long axis, $\mathrm{X}$ axis is the front-back direction of the bovine, and $\mathrm{Y}$ axis is the right-left direction of the bovine. Bone marrow contained in cancellous bone specimens was removed by using the oral irrigating device (OMRON). The specimens were frozen at $-20^{\circ} \mathrm{C}$ in normal saline and naturally defrosted for twelve hours before testing. In this study, the sizes of the specimens are expressed in terms of length of a side of a cube. Seven specimens were prepared for the compression test. After the compression test was conducted in the elastic region by the compression test method shown in 2.2 (as shown in Fig. 2), cubic specimens in $10 \mathrm{~mm}$ were polished to those cubic specimens in $5 \mathrm{~mm}$. After that, cubic specimens in $3 \mathrm{~mm}$ were made from those cubic specimens in $5 \mathrm{~mm}$ in the same way.

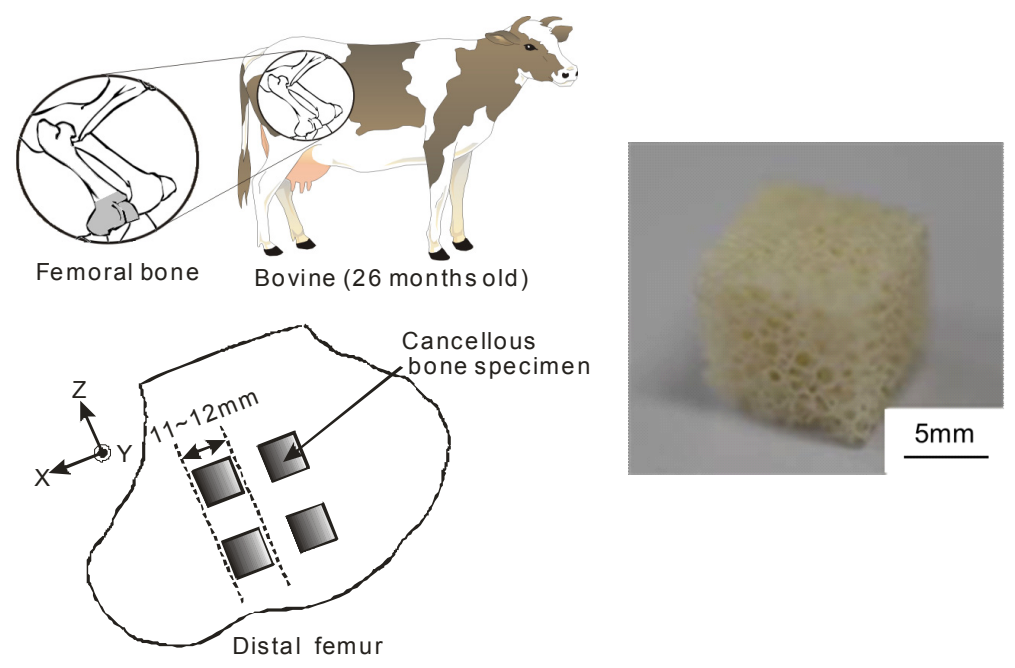

Figure 1: Cancellous bone specimens (cubic specimen in 10mm). 


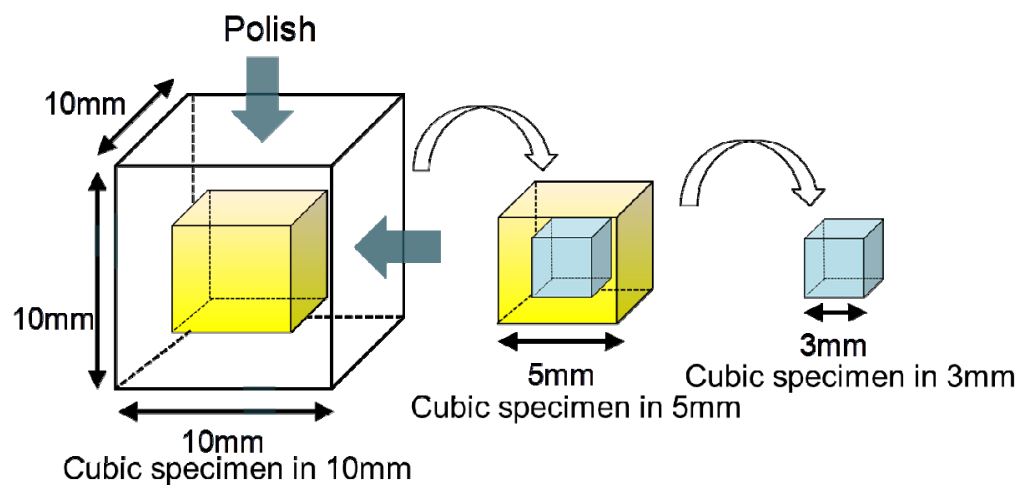

Figure 2: Manufacture method of specimen.

\subsection{Evaluation of Young's modulus}

In order to evaluate Young's modulus of cancellous bone specimens of each sizes, compression tests on $\mathrm{Z}$ axis direction were performed by using universal testing machine (Instron, 5566) with a load cell of $1 \mathrm{kN}$ at a crosshead speed of $0.0167 \mathrm{~mm} / \mathrm{sec}(1 \mathrm{~mm} / \mathrm{min})$. Crosshead speed was set based on JIS (JIS H 7902: Method for compressive test of porous metals). Fig. 3 shows an example of stress-strain curve of cancellous bone specimen. Young's modulus was calculated by using the linear part from (a) to (b) in Fig. 3.

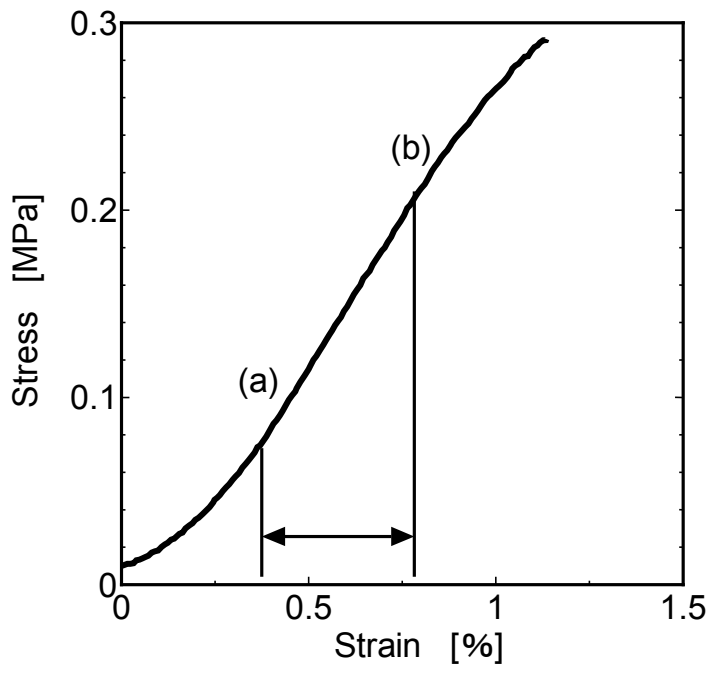

Figure 3: Stress-strain curve. 


\subsection{Evaluation of microstructural parameters}

As shown in Fig. 4, three-dimensional re-formation images (3D images) were obtained by using X-ray $\mu \mathrm{CT}$ (Shimadzu, SMX-160CTS) with a voxel size of $42 \mu \mathrm{m}$. To ensure consistent CT image resolution among all the datasets, the location of the scanner turntable was fixed at $105.0 \mathrm{~mm}$ in SOD and SID $347.8 \mathrm{~mm}$ in SID respectively. Microstructural parameters of 3D images of cubic specimen in $10 \mathrm{~mm}$, which were obtained before the compression test, were calculated by using analysis software (TRI/3D-BON, Ratoc). In addition, microstructural parameters of the specimen of $1-10 \mathrm{~mm}$ every $1 \mathrm{~mm}$ were calculated by using the value which was calculated from a point in the center of cubic specimen in $10 \mathrm{~mm}$. After the calculated parameters were normalized, standard deviation was calculated. Normalized microstructural parameters were obtained by calculating the average value of each microstructural parameter from 7 specimens. Microstructural parameters used in this study are fully described and abbreviated in table 1 and their abbreviations are shown.

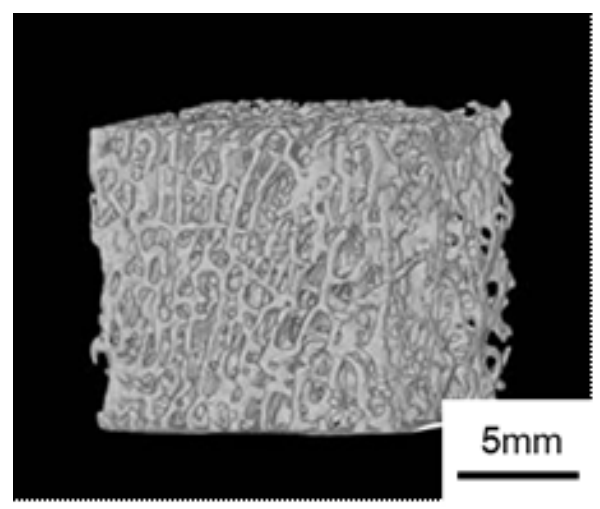

Figure 4: X-ray $\mu \mathrm{CT}$ image of cancellous bone specimen.

Table 1: Microstructural parameters.

\begin{tabular}{|c|c|}
\hline Abbrev. & Indices \\
\hline BV/TV $(\%)$ & Bone volume fraction \\
\hline BS/BV $\left(\mathrm{mm}^{-1}\right)$ & Bone surface/Bone volume \\
\hline Tb.Th $\left(\mathrm{mm}^{-1}\right.$ & Trabecular thickness \\
\hline Tb.N $\left(\mathrm{mm}^{-1}\right)$ & Trabecular number \\
\hline Tb.Sp $\left(\mathrm{mm}^{-1}\right)$ & Trabecular separation \\
\hline TBPf $\left(\mathrm{mm}^{-1}\right)$ & Trabecular Bone Pattern factor \\
\hline $\mathrm{DA}$ & Degree of anisotropy (MIL1/MIL3) \\
\hline MIL1 $(\mathrm{mm})$ & Mean Intercept Length 1 \\
\hline MIL3(mm) & Mean Intercept Length 3 \\
\hline
\end{tabular}




\subsection{Correlation coefficient}

After calculating the values of microstructural parameters and Young's modulus, correlation coefficient between microstructural parameters and Young's modulus was calculated. In this study, $\mathrm{R}^{2}$, which is generally used, is termed as correlation coefficient.

\section{Result and discussion}

\subsection{Correlation coefficients between Young's modulus and microstructural parameters}

As an example of the correlation coefficients between Young's modulus and microstructural parameters, Fig. 5 shows correlation coefficients between Young's modulus and BV/TV of cubic specimen in 5mm. Fig. 6 shows absolute values of correlation coefficients between Young's modulus and microstructural parameters of each cubic specimen size. Seven microstructural parameters, which were BV/TV, BS/TV, Tb.Th, Tb.N, TBPf, DA and MIL1 had the highest correlation coefficients between Young's modulus and cubic specimen in $5 \mathrm{~mm}$. Two microstructural parameters, which were Tb.Sp and MIL3 had the highest correlation coefficients between Young's modulus and cubic specimen in $3 \mathrm{~mm}$. In general, absolute value of the correlation coefficient is considered as follows if the value is 0.7 and over, it is highly correlated. Correlation coefficient of cubic specimen in $5 \mathrm{~mm}$ was 0.725 to 0.912 . BV/TV is a parameter representing the bone density. In addition, current clinical prediction of osteoporosis is based on BV/TV. As shown in the introduction, bone density was highly correlated with the strength. Therefore, it is considered to have been a valid result. Therefore, it is considered that cubic specimen in $5 \mathrm{~mm}$ is optimal for correlation coefficients among cubic specimen in $3 \mathrm{~mm}, 5 \mathrm{~mm}$ and $10 \mathrm{~mm}$.

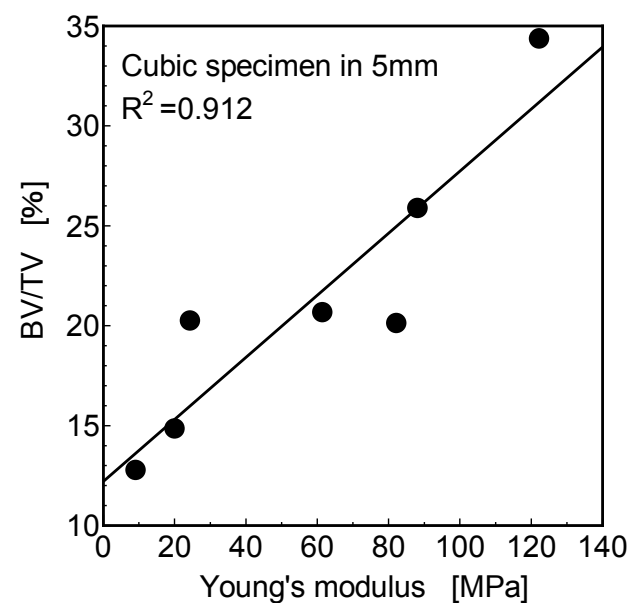

Figure 5: Correlation coefficients between Young's modulus and BV/TV. 


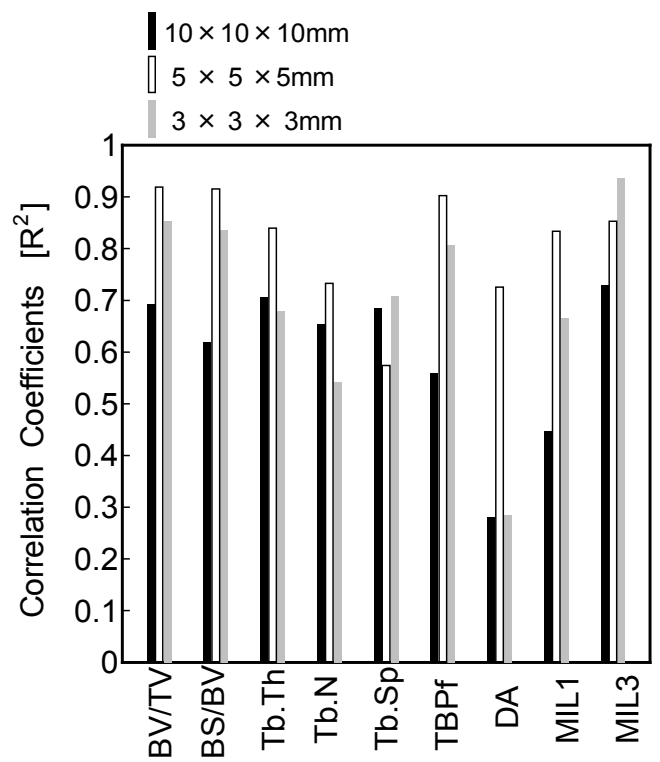

Microstructural parameters

Figure 6: Correlation coefficients between Young's modulus and microstructural parameters.

\subsection{Standard deviation of normalized microstructural parameters}

Fig. 7 shows standard deviation of normalized microstructural parameters. Microstructural parameters except for Tb.Sp and MIL3 had the smallest standard deviation when analysis region was in 4 or $5 \mathrm{~mm}$. On the other hand, standard deviation was relatively high when analysis region was in $1-3 \mathrm{~mm}$. Length per trabecular bone is around between several hundred $\mu \mathrm{m}$ and $2 \mathrm{~mm}$. In specimens region of $1-3 \mathrm{~mm}$, since the range which removed from cancellous bone becomes narrow, the difference in the amount of contained trabecular bone becomes large. Further, it is not possible to evaluate the orientation. Therefore, the standard deviations of specimens in $1-3 \mathrm{~mm}$ were large. From the above, it is considered that the variation in each microstructural parameter can be reduced by dividing cancellous bones into the blocks of 4 or $5 \mathrm{~mm}$.

These results showed that the specimen size of the lowered standard deviation was consistent with specimen region of higher correlation coefficient. 


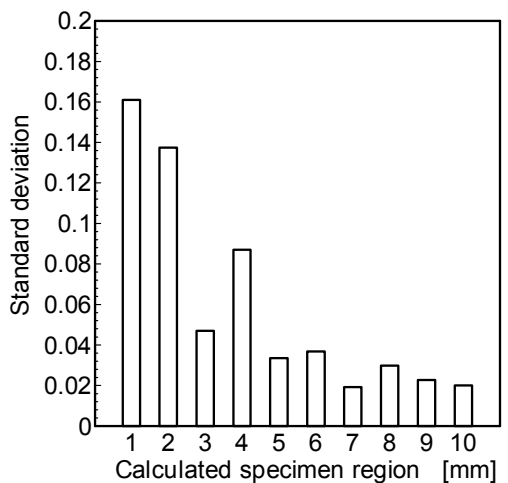

(e) Standard deviation of Tb.Sp

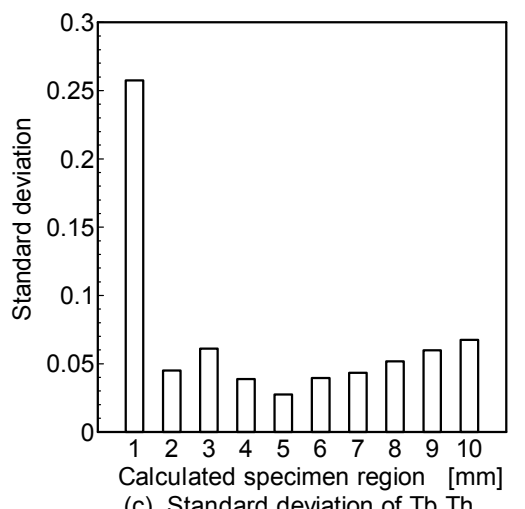

(c) Standard deviation of $\mathrm{Tb}$. Th

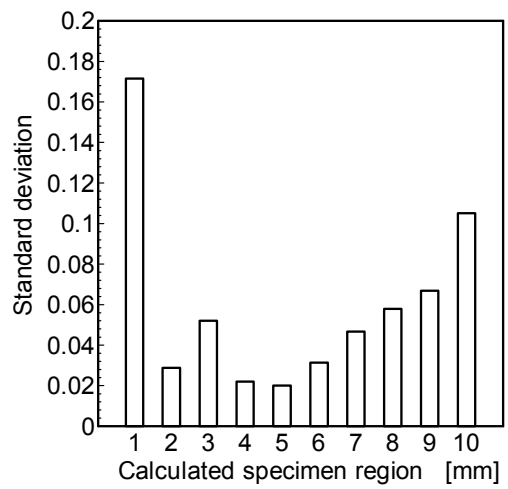

(g) Standard deviation of MIL1

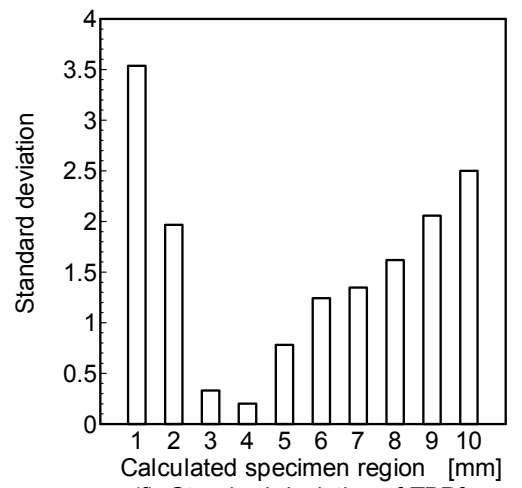

(f) Standard deviation of TBPf

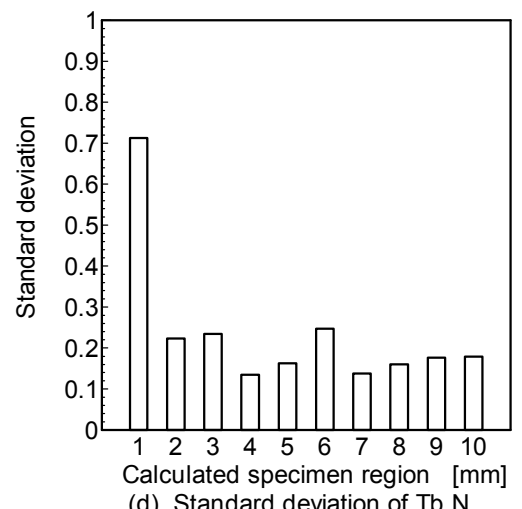

(d) Standard deviation of Tb.N

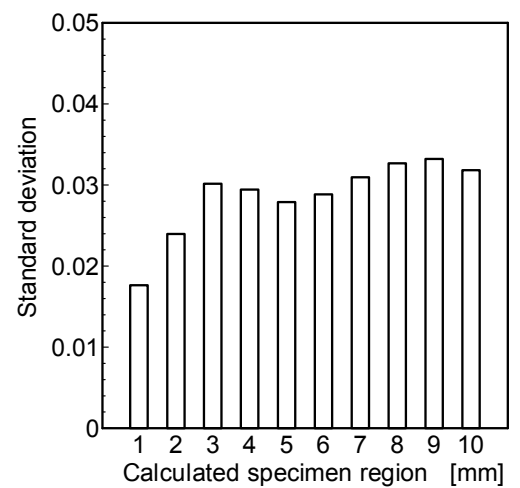

(h) Standard deviation of MIL3

Figure 7: Standard deviation of normalized microstructural parameters. 


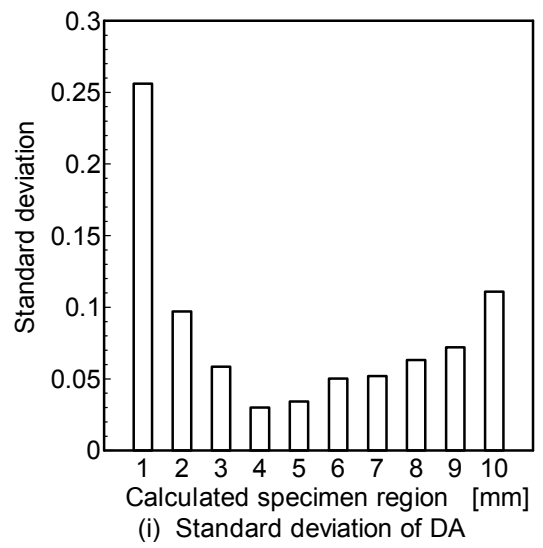

Figure 7: Continued.

\section{Conclusion}

In this study, the influence of the cancellous bone area on the correlation between the microstructural parameters, which were calculated by using X-ray microtomography images, and Young's modulus, obtained from the compression test, was clarified by targeting cancellous bone specimens of various regions, which were obtained from bovine femur. The following conclusions were obtained:

1. Cubic specimen in $5 \mathrm{~mm}$ had higher correlation between Young's modulus and microstructural parameters than cubic specimens in $3 \mathrm{~mm}$ and $10 \mathrm{~mm}$. Therefore, cancellous bone into the blocks of $5 \mathrm{~mm}$ can improve the correlation between the microstructural parameters and Young's modulus.

2. Standard deviation of microstructural parameters is reduced by dividing specimens into the blocks of 4 or $5 \mathrm{~mm}$. Therefore, cancellous bone into the blocks of 4 or $5 \mathrm{~mm}$ can be used to obtained uniform microstructural parameters.

\section{References}

[1] T. Nakamura, The Quality of Bone. J UOEH 19(2), pp. 157-164 (1997).

[2] N. Inoue, Fukugouzairyou to siteno hone, JCOM-36, p. 97 (2007).

[3] NIH Consensus Development Panel on Osteoporosis Prevention, Diagnosis, and Therapy, Osteoporosis prevention, diagnosis, and therapy, JAMA, 285, pp. 785-795 (2001).

[4] T. Sone, microstructure of bone and bone quality, THE BONE, Vol. 21, No. 1, pp. 35-39 (2007). 
[5] A. Nazarian, J. Muller, D. Zurakowski, Densitometric, morphometric and mechanical distributions in the human proximal femur, Journal of Biomechanics 40, pp. 2573-2579 (2007).

[6] S. Boyd, N. Shrive, G. Wohl, Measurement of cancellous bone strain during mechanical tests using a new extensometer device, Medical Engineering \& Physics 23, pp. 411-416 (2001).

[7] A. Burgers, J. Mason, G. Niebur, Compressive properties of trabecular bone in the distal femur, Journal of Biomechanics 41, pp. 1077-1085 (2008).

[8] E. Mittra, C. Rubin, B. Gruber, Evaluation of trabecular mechanical and microstructural properties in human calcaneal bone of advanced age using mechanical testing $\mu \mathrm{CT}$ and DXA, Journal of Biomechanics 41, pp. 368-375 (2008).

[9] E. Perlli, M. Baleani, C. Ohman, Dependence of mechanical compressive strength on local variations in microarchitecture in cancellous bone of proximal human femur, Journal of Biomechanics 41, pp. 438-446 (2008).

[10] F. Linde, I. Hvid, F. Madsen, The effect of specimen geometry on the mechanical behavior of trabecular bone specimens, Journal of Biomechanics, vol. 25, No. 4, pp. 359-368 (1992).

[11] L. Rogers, D. Mxoyle, Effect of specimen size on work-of-fracture measurements, Journal of Biomechanics, vol. 21, No. 11, pp. 919-926 (1988). 